\title{
Removal of Migrated Esophageal Stents in the Stomach Under the Guidance of Fluoroscopy: a Single-center Retrospective Study
}

Kewei Ren

Department of Interventional Radiology, The First Affiliated Hospital of Zhengzhou University Haitao Liu

Department of Interventional Radiology, The First Affiliated Hospital of Zhengzhou University

Zihe Zhou

Department of Interventional Radiology, The First Affiliated Hospital of Zhengzhou University

Yahua Li

Department of Interventional Radiology, The First Affiliated Hospital of Zhengzhou University

\section{Huibin Lu}

Department of Interventional Radiology, The First Affiliated Hospital of Zhengzhou University

Xinwei Han

Department of Interventional Radiology, The First Affiliated Hospital of Zhengzhou University

Jianzhuang Ren ( $\sim$ rjzjrk@126.com )

Department of Interventional Radiology, The First Affiliated Hospital of Zhengzhou University

\section{Research Article}

Keywords: Esophageal stent, migration, removal of stent, fluoroscopy guidance

Posted Date: March 6th, 2021

DOl: https://doi.org/10.21203/rs.3.rs-272800/v1

License: (c) (1) This work is licensed under a Creative Commons Attribution 4.0 International License. Read Full License 


\section{Abstract}

Background: Migrated esophageal self-expandable metal stents (SEMSs) increase the risk of bowel obstruction or perforation. The endoscopic removal of migrated stents is extremely difficult due to the inability to observe the distal end of the stent during retrieval. Here, we report our experience removing migrated esophageal stents in the stomach under the guidance of fluoroscopy.

Material and methods: The clinical data of patients with esophageal stents that migrated to the stomach between January 2016 and March 2020 were analyzed retrospectively. A total of 27 patients ( 9 females and 18 males) were included in this study. Three methods of retrieval were considered: direct removal via a fixed string, direct removal via a retrieval hook, and retrieval via guide wire and gooseneck snare.

Results: A total of 28 migrated esophageal stents in the stomachs of 27 patients were successfully removed under the guidance of fluoroscopy by the three methods mentioned above: 10 cases of direct removal via a fixed string, 14 cases of direct removal via a retrieval hook, and 3 cases of retrieval via a guide wire. The stent removal time was 18 (7-60) minutes. During the operation, one patient had a small amount of esophageal bleeding that was cured after symptomatic treatment, and one patient had a residual fracture stent wire that was removed under endoscopy.

Conclusion: The removal of migrated esophageal stents in the stomach under the guidance of fluoroscopy is a feasible and safe procedure.

\section{Background}

Esophageal stent placement is a safe and effective procedure performed to restore patency or occlude the fistula. Stent migration is a recognized complication of esophageal self-expandable metal stents (SEMSs). As a main complication, the rate of stent migration can reach $36 \%$ [1]. The distal migration rate is approximately $5.4 \%$, while the retention rate of stents in the stomach is approximately $3.7 \%$ [2]. Distal stent migration is rare, and the symptoms vary.

The most common symptom after stent migration is recurrent dysphagia. Other symptoms depend on the location of stent retention. Stents that migrate to the gastrointestinal tract can present worsening abdominal pain. However, migration into the colon can present constipation. Some cases can present without symptom. Although a distal migrated stent can pass through the pylorus and be excreted via the rectum, obstruction and perforation of the small bowel can also occur. To prevent severe complications secondary to distal migrated stents, it is recommended that migrated stents be removed whenever possible. Whether the migrated esophageal stent is removed through the mouth is still controversial. From the experience of De Palma et al [2], obstruction or perforation caused by stent migration is uncommon, and only a single case of 9 migrated stents leading to complications was reported.

Usually, an esophageal stent is removed under endoscopy. It is a challenging situation for endoscopists to remove a stent that has migrated to the stomach. A stent with a proximal lasso can be removed by 
grabbing and pulling the lasso with rat tooth forceps. However, it may be trickier to remove stents without a proximal lasso. Many methods have been attempted according to early reports [1]. The devices used included a double-channel endoscope, endoloops, a polypectomy snare and a biliary stent pusher. These devices are expected to reduce stent size to facilitate removal or reduce the diameter of the proximal stent to remove. However, the extraction of a migrated esophageal stent through endoscopy may be extremely difficult and is associated with a substantial risk of complications, including esophageal perforation and hemorrhage.

To remove esophageal stents that migrate to the stomach more safely and effectively, fluoroscopy guidance was used in this study. We aim to share our experience removing esophageal stents that migrated to the stomach under the guidance of fluoroscopy.

\section{Methods}

\section{Patients}

Informed consent was obtained from each patient.

Ethics committee approval was obtained for this retrospective study.

Patients undergoing retrieval of an esophageal stent that migrated to the stomach under the guidance of fluoroscopy between Jan 2016 and March 2020 were retrospectively enrolled. A total of 27 patients aged 5-93 years were enrolled in this study. All the diagnoses of 27 patients with esophageal stents that migrated to the stomach were confirmed by CT. The admission symptoms included recurring dysphagia in 13 cases and abdominal pain and vomiting in 5 cases. The other 9 cases were found by CT and had no classic symptoms. The general conditions and data of the patients are shown in Table 1. 
Table 1

General information of patients

\begin{tabular}{|ll|}
\hline Characteristic & Median (IQR) or No. (\%) \\
\hline Patients, (No.) & 27 \\
\hline Age, years & $70(5-93)$ \\
\hline Male/Female & $18 / 9(66.7 \% / 33.3 \%)$ \\
\hline Previous disease & \\
\hline Esophageal cancer & $12(44.4 \%)$ \\
\hline Cardiac cancer & $5(18.5 \%)$ \\
\hline Esophageal rupture & $6(22.2 \%)$ \\
\hline Esophageal stenosis & $3(11.1 \%)$ \\
\hline Esophago-tracheal fistula & $1(3.8 \%)$ \\
\hline Pathological results & \\
\hline Pathological results & $7(25.9 \%)$ \\
\hline Squamous carcinoma & $8(29.6 \%)$ \\
\hline Not & $12(44.5 \%)$ \\
\hline Indication of stent implantation & \\
\hline Dysphagia & $17(63.0 \%)$ \\
\hline Fistula & $8(29.6 \%)$ \\
\hline Esophageal stenosis & $2(7.4 \%)$ \\
\hline Symptoms of stent migration & \\
\hline Dysphagia & $13(48.2 \%)$ \\
\hline Abdominal pain and vomiting & $5(18.5 \%)$ \\
\hline None & $9(33.3 \%)$ \\
\hline Migration time (days) & $65-225)$ \\
\hline
\end{tabular}

\section{Procedure}

Method 1. Direct removal via a fixed string. These kinds of esophageal stents have a long nylon string loop on the proximal end. Long strings were used to fix the stent to prevent or monitor stent migration. After esophageal stent placement, the long string was passed through a nasoesophageal tube and then fixed. The long string was removed under the guidance of fluoroscopy 2 weeks post stenting. To remove esophageal stents that migrated to the stomach with a fixed sting, first, the fixed string was exchanged 
from the nasopharynx to the oropharynx. Then, a 12-14F sheath tube was introduced through the mouth along the fixed sting to the proximal end of the esophageal stent. Third, when the tip of the sheath tube reached the proximal end of the esophageal stent, the fixed string was pulled up to shrink the proximal end of the stent. The fixed string and sheath tube were withdrawn synchronously to remove the esophageal stent.

Method 2. Direct removal via a retrieval hook: First, a guide wire was introduced to the stomach with the cooperation of a catheter.

Then, the guide wire was adjusted to pass through the stent lumina, and the long sheath was exchanged. Third, a retrieval hook was introduced through the sheath to hook the stent. Finally, the retrieval hook, sheath, guide wire and esophageal stent were withdrawn together.

Method 3. Retrieval with a guide wire: First, a guide wire and catheter were introduced to the migrated stent via the mouth. Then, the long sheath was exchanged near the esophageal stent. Third, the guide wire and catheter were introduced through the luminal sheath, and the guide wire was adjusted to loop the center of the stent.

Next, a gooseneck snare was introduced to grasp the tip of the guide wire, and the stent was closely looped.

Finally, the sheath, gooseneck snare, guidewire and stent were withdrawn together.

\section{Results}

A total of 28 esophageal stents that migrated to the stomach in 27 patients were successfully removed under the guidance of fluoroscopy by the three methods mentioned above. Ten cases were removed by method 1 (Fig. 1). However, 15 esophageal stents in 14 patients were removed by method 2 (Fig. 2).

When the guide wire could not pass through the lumen of the stent after several attempts, method 3 was applied (Fig.

3). A total of 3 esophageal stents in 3 patients were retrieved by method 3 . All cases were definitively diagnosed by multislice computed tomography (MSCT) before the operation, and the operation was completed within one week after the definitive diagnosis. The operation mode, operation time and complications are listed in Table 2. 
Table 2

Operation-related information

\begin{tabular}{|ll|}
\hline Variable & Value \\
\hline Stent removal method & \\
\hline Method 1 & $10(37.0 \%)$ \\
\hline Method 2 & $14(51.9)$ \\
\hline Method 3 & $3(11.1 \%)$ \\
\hline Removal time (min) & $18(7-60)$ \\
\hline Complication & \\
\hline Residual metal wire of stent & 1 \\
\hline Esophageal bleeding & 1 \\
\hline
\end{tabular}

During the operation via method 2, one patient had a small amount of esophageal bleeding that was cured after symptomatic treatment. Additionally, via method 2, one patient had a residual fracture stent wire that was removed under gastroscopy.

After removal of the migrated stents, esophageal stents were reimplanted into 8 patients, 1 patient received radiotherapy, 5 patients received chemotherapy, 1 patient received surgical repair, and 12 patients received symptomatic support treatment.

\section{Discussion}

Most patients with esophageal cancer have reached the advanced stage when they are diagnosed, and surgical resection is not ideal. The 5-year survival rate is only $15-34 \%$ [3]. SEMS insertion provides a substantial advantage in the management of dysphagia in patients with malignant esophageal obstruction. SEMSs can quickly relieve dysphagia and occlude the fistula and has become the main palliative treatment for patients with advanced esophageal cancer. However, complications of stent implantation compromise the patient's quality of life. Stent migration is one of the major complications associated with esophageal stent implantations [4]. Most migration events occur within one month after stent placement [2]. After stent migration, dysphagia can recur, which seriously affects the quality of life of patients. If the stent migrates into the stomach or enters the intestinal tract, it can also cause serious complications such as perforation and intestinal obstruction. Karagul et al. believe that a stent left in the stomach will not cause related complications [5]. However, in this study, after stent migration, 2 patients suffered from abdominal pain and vomiting that may have been related to stent migration. More importantly, a stent left in the stomach is at an increased risk of migration to the intestinal tract, which is also problematic. According to previous reports $[1,2,6]$, the stent type, concurrent chemotherapy or radiotherapy, gastroesophageal junction tumors and sex are important factors for esophageal stent migration. Compared with bare metal self-expandable stents, covered stents are more helpful at 
preventing tumors from growing inward, but the transverse supporting force of covered stents is weak, and the friction between the covered stent and esophageal wall is small, so they are more likely to shift. Moreover, concerning malignant obstruction of the digestive tract, covered stents and bare metal stents have comparable technical success, clinical success, long-term patency and palliative treatment survival rates [7]. Some newly developed stents, such as partially covered stents, NiTi alloy double-layer stents, and fully covered self-expanding segmented metal stents [8, 9], have great advantages in preventing esophageal stent migration. Simultaneous radiotherapy and chemotherapy will reduce the volume of an esophageal tumor, and the stent will not fit well with the esophageal wall, which will increase the risk of esophageal stent migration. Tumors in the gastroesophageal junction include lower esophageal cancer and cardiac cancer. When a stent is placed here, the distal end of the stent has poor adhesion to the gastric mucosa, and some stents experience radial bending stress after placement; thus, these stents are more prone to migration. Therefore, patients with removable esophageal stents are carefully evaluated and monitored, and selective surgical removal of the migrated metal stents is advocated [10].

At present, most migrated esophageal stents are removed under endoscopy, which is often performed under general anesthesia or local anesthesia according to a few reports. For a stent that has migrated to the esophagus, the cable sleeve at the proximal end of the stent can be grasped by dental forceps under endoscopy so that the diameter of the proximal end of the stent can shrink and the stent can be pulled back. For stents without cable sleeves at the proximal end, a double-channel endoscope can be used to grasp the two sides of the proximal end of the stent and pull it out. For a stent that has migrated to the stomach, one with a cuff is easy to remove. When there is no proximal cuff, it is challenging and usually requires other devices, such as an inner ring, polypectomy snare, foreign body protection cover, or metal sheath tube [11-14]. When removing a stent in the stomach under endoscopy, there is a risk of insufficient stent contraction and esophageal injury. Some patients experience severe esophageal stenosis, which makes it difficult to remove the unseated stent. On the other hand, the working distance of the endoscope is limited, and a distal migrated stent cannot be removed under endoscopy. The fluoroscopic removal of esophageal stents is rarely reported for distal stent migration.

To solve the difficult problem of removing esophageal stents that have migrated to the stomach under endoscopy, we used fluoroscopy guidance. After esophageal stent placement, a stent withdrawal wire was exchanged from the nasal cavity and fixed. To improve the quality of life in the late stage, some patients will choose to have the stent withdrawal wire removed after the stent is stabilized.

In the absence of a stent removal wire, if a stent migrates to the stomach, the second method is needed; that is, a guide wire is passed through the stomach stent to facilitate the introduction of a sheath tube and a retrieved hook, and the force of the hook is located on the long axis of the stent.

To prevent damage to the tumor and new granulation tissue and prevent bleeding, the distal end of the stent is often hooked. During the removal process, the distal end of the stent will turn over into the stent so that the whole stent can be removed after turning over. When the length of the sheath is not enough or the angle with the stent cannot reach the distal end, the stent can be removed by hooking the proximal end, but the resistance will increase. At the same time, the possibility of tissue damage, bleeding and 
stent fracture will increase significantly. Sometimes, granulation tissue is wrapped at both ends of the detached stent.

In particular, for patients who have undergone gastrectomy, the stomach cavity is small, the guide wire cannot enter the stent cavity, and the stent cannot be removed by using a stent removal hook.

In these cases, the third method can be used.

When the sheath tube enters the stomach cavity, the guide wire is folded back and then enters the sheath tube so that the guide wire forms an annular catcher at the head end of the sheath tube, the middle position of the stent is captured by the annular catcher, and the guide wire is tightened to make the middle part of the stent smaller and then removed.

The fluoroscopic removal of migrated esophageal stents also has some complications, including esophageal bleeding, perforation, rupture and stent fracture. To avoid bleeding, perforation, etc., when removing a migrated stent, the operation should be performed quickly, and force is needed at the head end of the sheath instead of the esophageal wall when the stent is removed. The main cause of stent fracture is granulation tissue proliferation, which enters the stent lumen through the stent mesh. The removal of a stent 2 weeks after anastomotic leakage or 4 weeks after perforation may significantly reduce the complications related to stent use [15]. An esophageal stent will be endothelialized after 8 weeks, and it is not easy to remove after more than 10 months.

\section{Conclusion}

In summary, the removal of esophageal stents that have migrated to the stomach under the guidance of fluoroscopy is a feasible and safe procedure.

\section{Abbreviations}

SEMS: self-expanding metallic stent; DSA: digital subtraction angiography; MSCT: mult-slice spiral CT

\section{Declarations}

\section{Acknowledgements}

Not applicable.

\section{Authors' contributions}

RK, LY and HX were responsible for the conception and design of the study. LH, ZZ and LY were responsible for data collection and analysis. All authors read and approved the final version of the manuscript.

\section{Funding}

Not applicable. 
Availability of data and materials

The datasets used are available from the corresponding author on reasonable request.

\section{Ethics approval and consent to participate}

The study was approved by the Ethical Committee of the first affiliated hospital of Zhengzhou University. All methods were carried out in accordance with relevant guidelines and regulations. Written informed consent was obtained from all subjects or, if subjects are under 18, from a parent and/or legal guardian.

\section{Consent for publication}

Not applicable.

\section{Competing interests}

The authors declare that they have no competing interests.

\section{Author details}

${ }^{1}$ Department of Interventional Radiology, The First Affiliated Hospital of Zhengzhou University, Zhengzhou, China. ${ }^{2}$ Interventional Treatment and Clinical Research Center of Henan Province, Zhengzhou, China.

\section{References}

1. Martins BC, Retes FA, Medrado BF, et al. Endoscopic management and prevention of migrated esophageal stents [J]. World J Gastrointest Endosc, 2014; 6(2):49-54.

2. De Palma GD, lovino P, Catanzano C. Distally migrated esophageal self-expanding metal stents: wait and see or remove? [J]. Gastrointest Endosc, 2001, 53(1):96-98.

3. M. Sjoquist, B.H. Burmeister, B.M. Smithers, J.R. Zalcberg, R.J. Simes, A. Barbour, V. Gebski, A.G.-I.T. Group, Survival after neoadjuvant chemotherapy or chemoradiotherapy for resectable oesophageal carcinoma: An updated meta-analysis, Lancet Oncol. 12 (7) (2011) 681-692.

4. Mozafari Hozhabr, Dong Pengfei, Zhao Shijia et al. Migration resistance of esophageal stents: The role of stent design.[J] .Comput. Biol. Med., 2018, 100:43-49.

5. Karagul S, Yagci MA, Ara C, Tardu A, Ertugrul I, Kirmizi S, et al. Small bowel perforation due to a migrated esophageal stent: Report of a rare case and review of the literature. Int J Surg Case Rep. $2015 ; 11: 113-6$.

6. Pavlidis TE, Pavlidis ET. Role of stenting in the palliation of gastroesophageal junction cancer: A brief review. World J Gastrointest Surg. 2014 Mar 27; 6(3):38-4.

7. Yang Z, Wu Q, Wang F, Ye X, Qi X, Fan D. A systematic review and meta-analysis of randomized trials and prospective studies comparing covered and bare self-expandable metal stents for the treatment 
of malignant obstruction in the digestive tract. Int J Med Sci. 2013 Apr 27; 10(7):825-35.

8. Kim ES, Jeon SW, Park SY, Cho CM, Tak WY, Kweon YO, Kim SK, Choi YH. Comparison of doublelayered and covered Niti-S stents for palliation of malignant dysphagia. J Gastroenterol Hepatol. 2009 Jan; 24(1):114-9.

9. Bi Y, Ren J, Li J, Yu Z, Han X, Wu G. A novel fully covered self-expandable segmental metallic stents for the treatment of refractory esophageal stenosis. J Thorac Dis. 2019 Apr; 11(4):1363-1369.

10. Zhang W, Meng WJ, Zhou ZG. Multiple perforations of the jejunum caused by a migrated esophageal stent. Endoscopy. 2011; 43 Suppl 2 UCTN: E145-6.

11. Molina-Infante J, Fernandez-Bermejo M, Perez-Gallardo B. Removal of a migrated covered metallic stent through an esophageal stricture, with multiple endoloops. Endoscopy 2010; 42 Suppl 2:E268E269.

12. Raijman I, Marcon NE, Kandel G, Haber GB, Kortan P. Repositioning of an esophageal stent after migration using a snare. Gastrointest Endosc 1994; 40:652.

13. May A, Gossner L, Feess G, Bauer R, Ell C. Extraction of migrated self-expanding esophageal metal stents. Gastrointest Endosc 1999; 49: 524-526.

14. Jantsch H, Lechner G, Wittich GR, Wunderlich M, Karnel F,Niederle B. Dislocated Atkinson tubes: removal and repositioning with a balloon catheter. Radiology 1989; 170: 885-886.

15. De Ronde T, Martinet JP, Melange M. Easy removal of migrated self-expanding esophageal metal stent using an endoloop device. Gastrointest Endosc. 2000; 52(1):125-7.

\section{Figures}




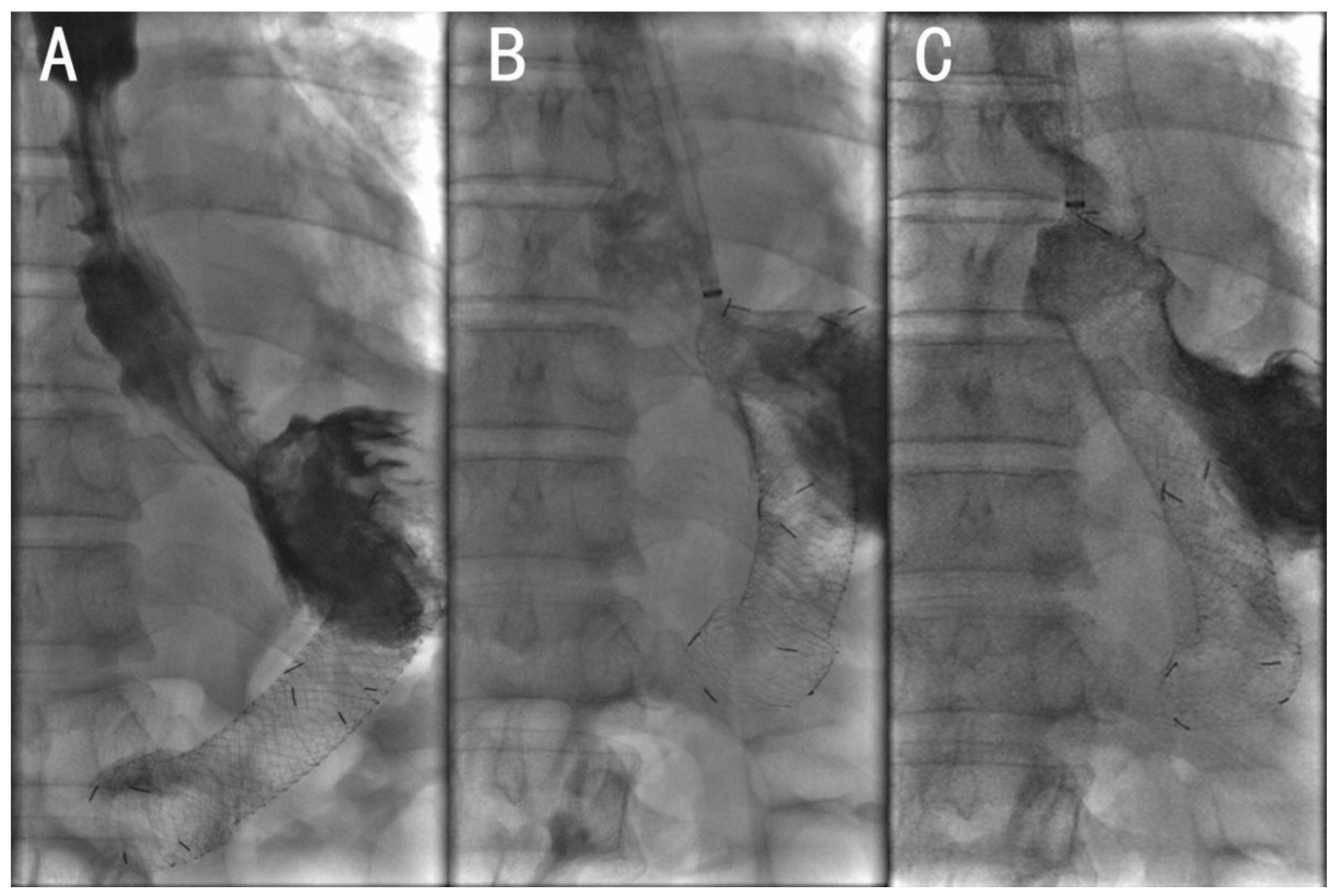

\section{Figure 1}

Direct removal via a fixed string. A. The migrated stent position was determined via fluoroscopy. B. A sheath tube was introduced through the fixed string, and the proximal end of the stent was supported. C. The fixed string was tightened to make the stent smaller, and the stent was removed.

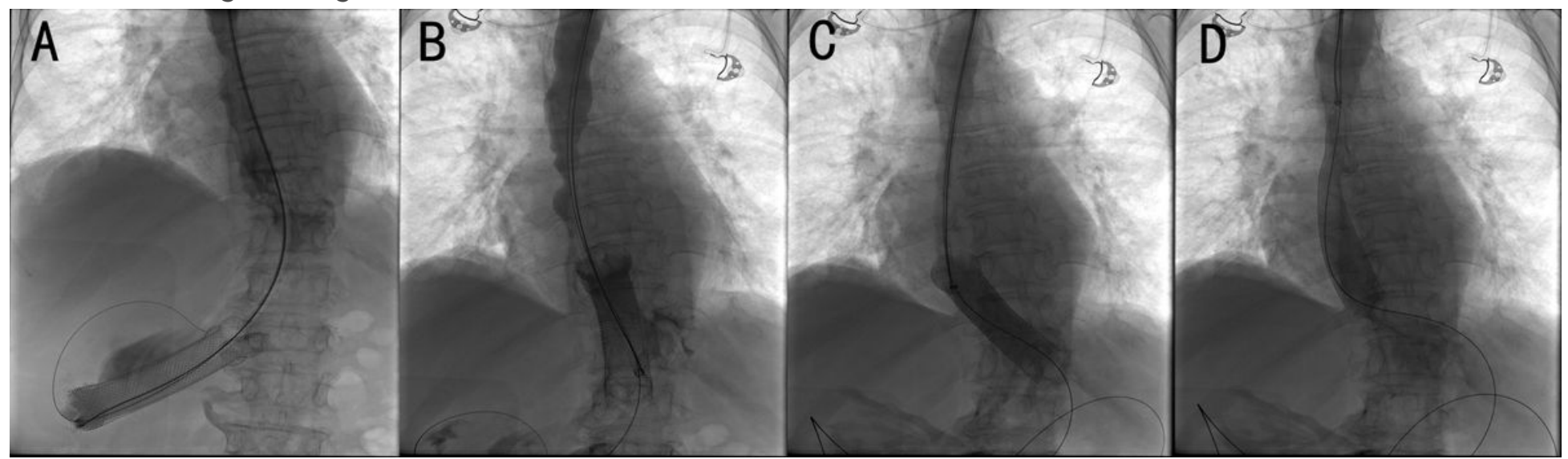

\section{Figure 2}

Direct removal via a retrieval hook. A. A guide wire, sheath tube and hook were transported to the distal end of the stent under the guidance of fluoroscopy. B. The distal end of the stent was lifted into the 
esophagus. C. A retrieval hook was used to grab the proximal end of the stent. D. The retrieval hook was tightened, and the stent was removed.

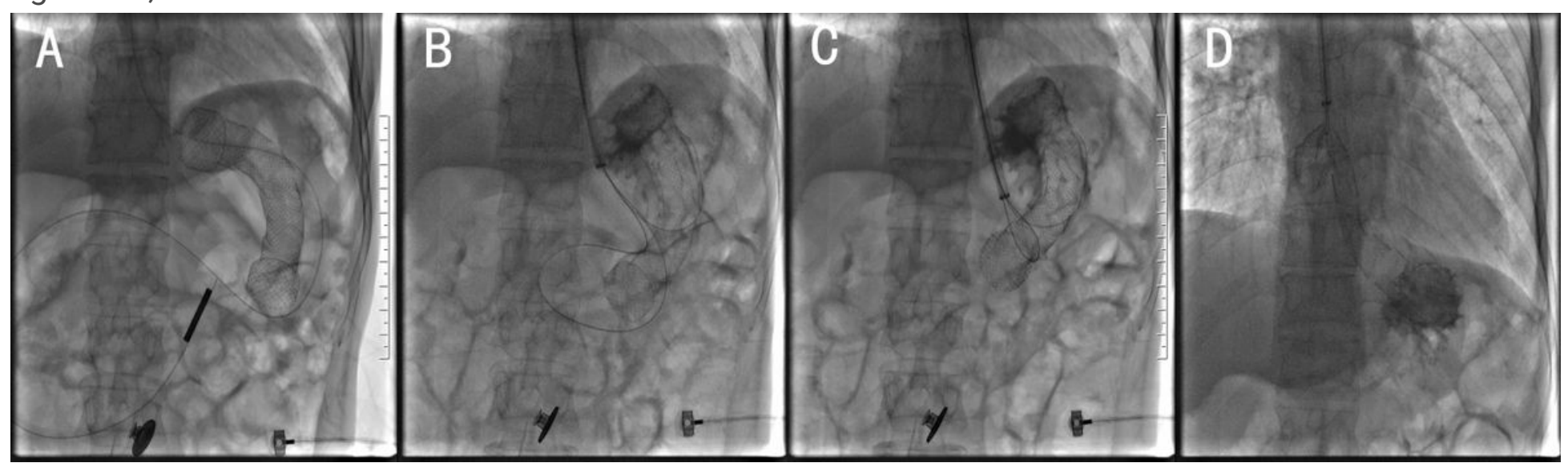

\section{Figure 3}

Retrieval via a guide wire. A. A guide wire was passed through the stent to the distal end. B. The end of the guide wire was grasped with a gooseneck snare to make the guide wire ring-shaped. $C$. The ring was adjusted to catch the migrated stent until placed. D. The migrated stent was successfully retrieved with a guide wire and gooseneck snare. 